INPLASY

PROTOCOL

To cite: Su et al. Reporting quality on interventional systematic review protocols for knee osteoarthritis: A systematic review plan. Inplasy protocol 202110111. doi: 10.37766/inplasy2021.1.0111

Received: 28 January 2021

Published: 28 January 2021

Corresponding author: Yaohu Su

550114673@qq.com

Author Affiliation: Gansu University of Chinese Medicine

\section{Support: 18JR2FA009.}

Review Stage at time of this submission: Formal screening of search results against eligibility criteria.

Conflicts of interest: None.

\section{Reporting quality on interventional systematic review protocols for knee osteoarthritis: A systematic review plan}

\author{
Su, YH¹; Wang, MW².
}

Review question / Objective: 1. How about the reporting quality of interventional systematic review protocols for knee osteoarthritis? 2. What are the potential factors influencing the reporting quality of interventional systematic review protocols for knee osteoarthritis?

Condition being studied: Knee osteoarthritis is one of the most prevalent chronic degenerative joint tissues, bothering the quality of life of suffers, and leading to huge social and economic burden. Protocol can reduce the selection reporting of systematic reviews as the key tool to synthesize all available evidence related to a special question. Recently, many interventional systematic review protocols for knee osteoarthritis has been published in several journals, but their reporting quality remains unknown. Moreover, through identifying the influencing factors for reporting quality can help researchers to improve the reporting quality of interventional systematic review protocols for knee osteoarthritis.

INPLASY registration number: This protocol was registered with the International Platform of Registered Systematic Review and Meta-Analysis Protocols (INPLASY) on 28 January 2021 and was last updated on 28 January 2021 (registration number INPLASY202110111).

\section{INTRODUCTION}

Review question / Objective: 1.How about the reporting quality of interventional systematic review protocols for knee osteoarthritis? 2.What are the potential factors influencing the reporting quality of interventional systematic review protocols for knee osteoarthritis?
Condition being studied: Knee osteoarthritis is one of the most prevalent chronic degenerative joint tissues, bothering the quality of life of suffers, and leading to huge social and economic burden. Protocol can reduce the selection reporting of systematic reviews as the key tool to synthesize all available evidence related to a special question. Recently, 
many interventional systematic review protocols for knee osteoarthritis has been published in several journals, but their reporting quality remains unknown. Moreover, through identifying the influencing factors for reporting quality can help researchers to improve the reporting quality of interventional systematic review protocols for knee osteoarthritis.

\section{METHODS}

Search strategy: The interventional systematic review protocols for knee osteoarthritis will be searched via PubMed and Embase databases for all papers published up to February 2021. The key search terms will be used such as: "Osteoarthritis, Knee", "knee osteoarthritis", "knee osteoarthritides", "knee osteoarthrosis", "knee osteoarthroses", "knee arthritis", "degenerative knee", "review", "meta*", "protocol*", "plan*", etc. Meanwhile, the references' checklist of included protocols will be checked to find the potential articles.

Participant or population: Population with knee osteoarthritis, there will be no other restrictions such as age, gender, race, or nationality.

Intervention: All available treatments mentioned in the included protocols, such as traditional therapies (herbs, massage, etc.), drug and surgery.

Comparator: Similar to the abovementioned in Intervention(s), exposure(s).

Study designs to be included: We will include the interventional systematic review protocols for knee osteoarthritis.

Eligibility criteria: We will include interventional systematic review protocols for knee osteoarthritis published in English. A systematic review refers to "A review of a clearly formulated question that uses systematic and explicit methods to identify, select and critically appraise relevant research, and to collect and analyze data from the studies included in the review".
Population with knee osteoarthritis was confirmed by clinician' judgement based on clinical manifestations, imaging examination and other tests. All available treatments [such as traditional therapy (e.g., herbs, massage, short-wave), drug (e.g., IA hyaluronic acid, prescription chondroitin sulfate), surgery and other interventions, e.g., stem cell therapy] mentioned in the included systematic review protocols will be considered by this present study. All available outcomes mentioned in the included protocols will be considered, they could be efficacy (e.g., pain reduction and quality of life improvement), safety (i.e., any unexpected complications), and economics (e.g., cost in hospital) data. The other publications will be excluded.

Information sources: PubMed and Embase databases.

Main outcome(s): All available outcomes mentioned in the included protocols, they could be safety, efficacy and economics data.

Quality assessment / Risk of bias analysis: Two independent reviewers will apply the PRISMA-P checklist to assess the included protocols of interventional systematic review for knee osteoarthritis, and any disagreements will be resolved by discussion. Three options including "Yes," "Partial Yes," or "No" will be used to complete the reporting quality assessment, and "1", " 0.5 ", and "0" point will be scored for each option, respectively

Strategy of data synthesis: The general information reported in the included protocols will be summarized descriptively. For the compliance of PRISMA-P checklist, frequencies with percentage will be used and total scores for each included protocol will be summed. The univariable and multivariable linear regression will be conducted to exploring the potential influencing factors [methodologist involvement (Yes vs. No), number of authors ( $\geq$ the median vs. the median), international cooperation (Yes vs. No), registration website (PROSPERO vs. 
others, e.g., OSF and INPLASY), funding information (Yes vs. No+Not reported)] for the reporting quality of included protocols. All results will be presented using the simple table, radar chart, and forest plot. Stata 16 and Excel 2016 will be used to manage and analyze data.

Subgroup analysis: Not applicable.

Sensitivity analysis: Not applicable.

Language: English.

Country(ies) involved: China.

Keywords: Knee osteoarthritis, Systematic review, Reporting quality, PRISMA-P, Protocols.

Contributions of each author:

Author 1 - Yaohui Su.

Email: 550114673@qq.com

Author 2 - Mingwang Wang.

Email: zmw2006@126.com 